\title{
Relationship of Catastrophizing to Fatigue Among Women Receiving Treatment for Breast Cancer
}

\author{
Paul B. Jacobsen \\ University of South Florida
}

\author{
Michael A. Andrykowski \\ University of Kentucky College of Medicine
}

\author{
Christina L. Thors \\ University of South Florida
}

\begin{abstract}
This study examined the relationship of catastrophizing to fatigue in 80 women receiving chemotherapy (CT) or radiotherapy (RT) for treatment of early stage breast cancer. Findings revealed expected relationships between catastrophizing and fatigue among women receiving RT but not CT. Among RT patients, those high in catastrophizing reported significantly greater fatigue than was reported by those low in catastrophizing; among CT patients, differences in fatigue based on level of catastrophizing were not significant. Likewise, catastrophizing was found to account for significant variability in subsequent reports of fatigue among RT patients but not CT patients. These findings extend research on catastrophizing beyond previously studied relationships with pain and are consistent with the view that, as the inherent symptom-producing potential of treatment decreases, psychological factors play a greater role in patients' experience of symptoms.
\end{abstract}

Fatigue has been described as the most prevalent symptom of cancer treatment (Winningham et al., 1994). This assertion is supported by surveys indicating that more cancer patients report fatigue during treatment than report other common symptoms such as pain or nausea (Greene, Nail, Fieler, Dudgeon, \& Jones, 1994; Longman, Braden, \& Mishel, 1996). Although common, the experience of treatment-related fatigue varies considerably from patient to patient (Berger 1998; Jacobsen, Hann, et al., 1999; Smets et al., 1998).

The current study examined the possibility that reliance on catastrophizing might explain differences in fatigue among patients undergoing cancer treatment. Catastrophizing has been defined as a cognitive process characterized by a lack of confidence and an expectation of negative outcomes (Sullivan \& D'Eon, 1990). We chose to focus on catastrophizing for two reasons. First, a considerable body of research has documented a relationship between catastrophizing and the experience of pain, another symptom with aversive properties (Sullivan et al., 2001). Second, preliminary evidence suggests a relationship between catastrophizing

Paul B. Jacobsen and Christina L. Thors, Department of Psychology, University of South Florida; Michael A. Andrykowski, Department of Behavioral Science, University of Kentucky College of Medicine.

This research was supported in part by National Cancer Institute Grant R01 CA82822. We thank Simon Wessely for his consultation on study design, Krista Varkey for her assistance with data collection, and Kristine Donovan and Christine Marsella for their assistance with manuscript preparation.

Correspondence concerning this article should be addressed to Paul B. Jacobsen, Department of Psychology (PCD 4118), University of South Florida, 4202 East Fowler Avenue, Tampa, FL 33620. E-mail: jacobsen@ moffitt.usf.edu and the experience of fatigue in cancer patients. Previous research has shown that greater use of catastrophizing is associated with more severe fatigue in women who had completed chemotherapy (CT) for breast cancer (Broeckel, Jacobsen, Horton, Balducci, \& Lyman, 1998) as well as with more severe and disruptive fatigue in women who were undergoing or had completed bone marrow transplantation, CT, or radiotherapy (RT) for breast cancer (Jacobsen, Azzarello, \& Hann, 1999). To the best of our knowledge, these are the only studies to have examined the relation of catastrophizing to fatigue in cancer patients.

The current study was designed to address several limitations of the prior research. One limitation was the use of a cross-sectional study design, which does not permit a consideration of the temporal predictive value of catastrophizing in relation to fatigue. A second limitation was the absence of a sample restricted to women currently undergoing treatment for breast cancer. This feature obscures an understanding of the importance of catastrophizing during the active treatment period when fatigue may be of greater intensity (Geinetz et al., 2001; Irvine, Vincent, Graydon, \& Bubela, 1998). A third limitation was the inclusion of women receiving different types of treatment in insufficient numbers to perform adequate tests of possible treatment-related differences in the relationship between catastrophizing and fatigue. This issue merits examination because studies of women who have completed treatment for early stage breast cancer suggest that administration of CT may result in greater fatigue than administration of RT (Bower et al., 2000; Woo, Dibble, Piper, Keating, \& Weiss, 1998).

The current study addressed these limitations through the use of a prospective longitudinal design in which similar numbers of women with early stage breast cancer scheduled to receive CT or RT were assessed before the start of treatment and, again, at the 
end of treatment. The primary aim of the study was to test the hypothesis that greater use of catastrophizing in response to fatigue would be related to greater fatigue severity and disruptiveness. Additional aims were to identify the ability of catastrophizing to predict subsequent reports of fatigue and to examine the possible interactive effects of type of treatment and level of catastrophizing on the experience of fatigue. With regard to predictive ability, we sought to determine the degree to which catastrophizing at treatment onset explained variability in subsequent fatigue by controlling for fatigue at treatment onset. With regard to interactive effects, we sought to determine whether the impact of catastrophizing differed by type of treatment. On the basis of prior research (Andrykowski \& Gregg, 1992), we speculated that a psychological variable such as catastrophizing might play a greater role in the experience of fatigue among patients receiving the less inherently fatiguing of the two treatments (i.e., RT).

\section{Method}

Participants were women scheduled to be treated with CT or RT for early stage breast cancer at the Moffitt Cancer Center (Tampa, FL) or the Markey Cancer Center (Lexington, KY). Eligibility criteria were that participants (a) be at least 18 years of age, (b) have no documented or observable psychiatric or neurological disorders that would interfere with study participation (e.g., dementia, psychosis), (c) be able to speak and read standard English, (d) have no history of cancer other than basal-cell skin carcinoma, (e) be diagnosed with Stage 0,1 , or 2 breast cancer, (f) have been treated surgically with lumpectomy or mastectomy, $(\mathrm{g})$ be scheduled to receive a minimum of four cycles of CT following surgery or be scheduled to receive RT following surgery, (h) have no prior history of treatment with either CT or RT, (i) have no other chronic or life-threatening diseases in which fatigue is a prominent symptom (e.g., AIDS, multiple sclerosis, chronic fatigue syndrome), and (j) sign an informed consent form approved by the University of South Florida or the University of Kentucky College of Medicine.

Eligibility was determined by chart review and consultation with the attending physician. Those women who provided informed consent completed a pretreatment questionnaire on the day of their first clinic visit for CT or RT. A follow-up questionnaire was administered during the last scheduled visit for CT or RT. Of 100 women considered eligible and asked to participate, 90 accepted. Following consent, 8 women (9\%) became ineligible (because of changes in treatment plans or a decision to complete treatment elsewhere) and 2 (2\%) elected to discontinue participation before completing the follow-up assessment. All subsequent analyses are based on the 80 women who provided both baseline and follow-up data.

Demographic data were obtained at the pretreatment assessment through use of a standard self-report questionnaire. We reviewed medical charts to obtain information about disease and treatment characteristics. We assessed fatigue at both the pretreatment and follow-up assessments with the Fatigue Symptom Inventory (Hann et al., 1998). As in prior research (Broeckel et al., 1998), analyses focused on items assessing fatigue severity and fatigue disruptiveness. Participants rated their average level of fatigue severity in the past week on an 11-point scale that ranged from 0 (not at all fatigued) to 10 (as fatigued as I could be). They rated perceived disruptiveness on 11-point scales that ranged from 0 (no interference) to 10 (extreme interference) and that asked about the degree to which fatigue interfered with general level of activity, ability to bathe and dress, normal work activity, ability to concentrate, relations with others, enjoyment of life, and mood in the past week. These ratings were summed to yield a total disruptiveness score ( $\alpha$ s ranged from .92 to .94). Previous research has demonstrated the reliability and validity of Fatigue Symptom Inventory severity and disruptiveness scores in women with breast cancer (Broeckel et al., 1998; Hann et al., 1998). Catastrophizing was assessed at the pretreatment assessment using the 10-item Fatigue Catastrophizing Scale (FCS; Jacobsen, Azzarello, \& Hann, 1999). Respondents rated on 5-point scales $(1=$ never true to $5=$ all of the time true $)$ how often each item is true for them when they have experienced fatigue (e.g., "I find myself expecting the worst when I'm fatigued"). We derived a total score by computing the mean of the 10 ratings $(\alpha=.85)$. Previous research has shown that higher scores on the FCS are associated with concurrent reports of more severe fatigue in women who were undergoing or had completed treatment for breast cancer (Broeckel et al., 1998; Jacobsen, Azzarello, \& Hann, 1999).

\section{Results}

The 80 participants ranged in age from 32 to 78 years $(M=$ $55.3 ; S D=10.0)$. The majority were White $(95 \%)$, married $(83 \%)$, and reported a household income of at least $\$ 40,000$ per year (64\%). Forty-one percent were college graduates and 67\% were postmenopausal at treatment initiation. Seven percent had Stage 0 disease, 53\% had Stage 1 disease, and $40 \%$ had Stage 2 disease. Eighty-two percent had undergone lumpectomy, $14 \%$ had undergone mastectomy, and $4 \%$ had undergone both lumpectomy and mastectomy. Fifty-three percent received CT and $47 \%$ received RT during the course of the study. With one exception (a patient who received $309 \mathrm{mg}$ of doxorubicin and no cyclophosphamide), all CT patients received a regimen that included both doxorubicin (range: 368-562 mg) and cyclophosphamide (range: 3,640-5,500 mg). With one exception (a patient who received 3,900 cGy of radiation over 15 treatments), all RT patients received between 5,000 and 6,640 cGy of radiation over 25 to 36 treatments.

Preliminary analyses indicated that women treated with RT were significantly older $(M=58.5, S D=9.7)$ than women treated with CT $(M=52.5, S D=9.7 ; p<.01)$. Because of the inherent natures of $\mathrm{CT}$ and $\mathrm{RT}$, the interval between the pretreatment and follow-up assessments also differed significantly between treatment groups. This interval averaged 42.5 days $(S D=8.9$; range: $20-61)$ for the RT group versus 100.5 days $(S D=44.3$; range: 61-187) for the CT group ( $p<.001)$. Also as expected, women treated with RT were significantly more likely to have undergone lumpectomy than mastectomy ( $p=.001)$ and to have earlier stage disease $(p=.001)$ than women treated with CT (see Table 1). The groups did not differ significantly with regard to race and/or ethnicity, marital status, education, income, or menopausal status ( $p$ s $>.05)$.

We conducted correlational analyses to examine the relation of demographic and clinical variables to catastrophizing and fatigue severity and disruptiveness. As shown in Table 2, the only demographic or clinical variable to yield a significant correlation was educational level. Specifically, a lower educational level was associated with greater fatigue severity and disruptiveness at the pretreatment assessment $(p<.05)$. Educational level also demonstrated a marginally significant negative correlation with fatigue severity $(p=.08)$ and disruptiveness $(p=.07)$ at the follow-up assessment

To examine the impact of catastrophizing on fatigue and explore possible interactive effects between catastrophizing and type of treatment, we entered patients' ratings of their average fatigue severity for the past week into a 2 (time: pretreatment and followup) $\times 2$ (treatment: CT or RT) $\times 2$ (catastrophizing: low or high) repeated measures analysis of variance design. FCS scores were dichotomized into high and low groups on the basis of the sample 
Table 1

Relation of Demographic and Clinical Variables to Treatment Group

\begin{tabular}{|c|c|c|c|c|c|}
\hline \multirow[b]{3}{*}{ Variable } & \multicolumn{4}{|c|}{ Group } & \multirow[b]{3}{*}{$p^{\mathrm{a}}$} \\
\hline & \multicolumn{2}{|c|}{ Chemotherapy } & \multicolumn{2}{|c|}{ Radiotherapy } & \\
\hline & $n$ & $\%$ & $n$ & $\%$ & \\
\hline \multicolumn{6}{|l|}{ Race/ethnicity } \\
\hline White & 39 & 49 & 37 & 46 & .36 \\
\hline Non-White & 3 & 4 & 1 & 1 & \\
\hline \multicolumn{6}{|l|}{ Marital status } \\
\hline Married & 35 & 43 & 31 & 39 & .84 \\
\hline Not married & 7 & 9 & 7 & 9 & \\
\hline \multicolumn{6}{|l|}{ Education } \\
\hline College graduate & 20 & 25 & 13 & 16 & .22 \\
\hline Non-college graduate & 22 & 28 & 25 & 31 & \\
\hline \multicolumn{6}{|l|}{ Household Income } \\
\hline Over $\$ 40,000$ & 28 & 38 & 23 & 33 & .57 \\
\hline Under $\$ 40,000$ & 10 & 14 & 11 & 15 & \\
\hline \multicolumn{6}{|l|}{ Menopausal status } \\
\hline Pre- or perimenopausal & 17 & 22 & 9 & 11 & .13 \\
\hline Postmenopausal & 25 & 32 & 28 & 35 & \\
\hline \multicolumn{6}{|l|}{ Surgery type } \\
\hline Lumpectomy only & 28 & 35 & 38 & 48 & .001 \\
\hline Mastectomy & 14 & 17 & 0 & 0 & \\
\hline \multicolumn{6}{|l|}{ Disease stage } \\
\hline Stage 0 or 1 & 12 & 15 & 36 & 45 & .001 \\
\hline Stage 2 & 30 & 38 & 2 & 2 & \\
\hline
\end{tabular}

Note. Eight participants declined to provide information about income, and 1 participant declined to provide information about menopausal status.

${ }^{a}$ The $p$ values are for chi-square tests or Fisher's exact tests of association.

median $(<1.3=$ low; $>1.4=$ high $)$. The resulting groups differed significantly in level of catastrophizing, $t(78)=11.75$, $p<.0001$. Education was included as a covariate on the basis of its observed relation to fatigue severity. With regard to main effects (see Table 3$)$, significant results $(p<.05)$ were obtained for time, treatment, and catastrophizing. With regard to interaction effects, there was no evidence of a significant $(p<.05)$ Time $\times$ Treatment $\times$ Catastrophizing interaction or a Time $\times$ Treatment interaction. However, as shown in Table 3, catastrophizing did interact with treatment to influence the level of fatigue severity $(p<.001)$.

The Treatment $\times$ Catastrophizing interaction is illustrated in Figure 1. Visual inspection of mean scores and simple effects analyses indicated that, among patients administered CT, there was little difference in fatigue severity on the basis of level of catastrophizing, $F(1,76)=0.14, p>.05$. In contrast, among patients administered RT, those high in catastrophizing reported more severe fatigue than those low in catastrophizing, $F(1,76)=13.21$, $p<.001$. Additional comparisons indicated that fatigue severity in RT patients low in catastrophizing was less than that reported by CT patients either low or high in catastrophizing, $F(1,76)=8.06$, $p<.01$, and $F(1,76)=10.21, p<.01$. However, fatigue severity in RT patients high in catastrophizing did not differ from that reported by $\mathrm{CT}$ patients either low or high in catastrophizing, $F(1$, $76)=0.74, p>.05$, and $F(1,76)=0.24, p>.05$.

The same $2 \times 2 \times 2$ repeated measures analysis of variance, with education included as a covariate, was conducted using patients' fatigue disruptiveness scores as the dependent variable. As with fatigue severity, there were significant main effects $(p<.05)$ for time, treatment, and catastrophizing and a significant interaction $(p<.05)$ between treatment and catastrophizing (see Table $4)$. The pattern of mean differences corresponding to these significant effects was similar to that observed for fatigue severity.

Hierarchical regression analyses were conducted to identify the ability of catastrophizing to predict follow-up levels of fatigue severity and disruptiveness. We entered pretreatment fatigue scores into the equations in Step 1 to transform the dependent variables into residualized change scores, then followed by entering pretreatment catastrophizing scores in Step 2. In light of the significant Treatment $\times$ Catastrophizing interactions reported previously, separate analyses were conducted for CT and RT patients. Among CT patients, catastrophizing did not explain additional significant variability in follow-up fatigue severity or disruptiveness scores. After accounting for pretreatment levels, catastrophizing among CT patients accounted for $1 \%$ of the variability in fatigue severity at follow-up ( $p=.64)$ and $1 \%$ of the variability in fatigue disruptiveness at follow-up $(p=.61)$. In contrast, catastrophizing explained significant additional variability in

Table 2

Relation of Demographic and Clinical Variables to Catastrophizing and Fatigue Severity and Disruptiveness

\begin{tabular}{|c|c|c|c|c|c|}
\hline \multirow[b]{2}{*}{ Variable } & \multirow[b]{2}{*}{ Catastrophizing } & \multicolumn{2}{|c|}{ Pretreatment fatigue } & \multicolumn{2}{|c|}{ Follow-up fatigue } \\
\hline & & Severity & Disruptiveness & Severity & Disruptiveness \\
\hline Age & -.17 & -.05 & -.10 & -.05 & -.09 \\
\hline Educational level & -.02 & $-.25 *$ & $-.22 *$ & -.20 & -.20 \\
\hline Income level & .15 & -.06 & -.13 & -.12 & -.09 \\
\hline Race/ethnicity (White $=0$ /other $=1$ ) & -.03 & -.17 & -.15 & -.11 & -.11 \\
\hline Marital status $($ married $=0 /$ other $=1$ ) & .07 & -.03 & .10 & .11 & .11 \\
\hline Menopausal status (pre- or perimenopausal $=0$ / & & & & & \\
\hline postmenopausal $=1)$ & -.09 & -.01 & -.03 & -.04 & .02 \\
\hline Surgery type (lumpectomy $=0 /$ mastectomy $=1$ ) & -.11 & .07 & -.01 & .03 & .04 \\
\hline Disease stage $(0,1=0 / 2=1)$ & -.15 & -.01 & -.01 & .02 & .02 \\
\hline Treatment type $($ chemotherapy $=0 /$ radiotherapy $=1$ ) & .04 & -.16 & -.19 & -.12 & -.19 \\
\hline Recruitment location $($ Kentucky $=0$, Florida $=1)$ & .01 & .19 & .19 & .08 & .05 \\
\hline
\end{tabular}

Note. The $p$ values are for point-biserial or Pearson's correlation coefficients.

$* p<.05$. 
Table 3

Repeated Measures Analysis of Variance of Fatigue Severity Scores

\begin{tabular}{lrrrr}
\hline \multicolumn{1}{c}{ Effect } & $d f$ & SS & MS & $F$ \\
\hline Between subjects & & & & \\
$\quad$ Treatment & 1 & 21.83 & 21.83 & $4.23^{*}$ \\
Catastrophizing & 1 & 38.26 & 38.26 & $7.42^{* *}$ \\
$\quad$ Treatment $\times$ Catastrophizing & 1 & 24.89 & 24.89 & $4.83^{*}$ \\
$\quad$ Residual & 75 & 386.68 & 5.16 & \\
Within subject & 1 & 38.26 & 38.26 & $18.70^{* * *}$ \\
Time & 1 & 0.00 & 0.00 & 0.00 \\
Time $\times$ Catastrophizing & 1 & 0.28 & 0.28 & 0.14 \\
Time $\times$ Treatment & 1 & 3.31 & 3.31 & 1.62 \\
Time $\times$ Catastrophizing $\times$ Treatment & 75 & 153.43 & 2.05 & \\
$\quad$ Residual & & & & \\
\hline
\end{tabular}

Note. $\mathrm{SS}=$ sums of squares; $\mathrm{MS}=$ mean square.

$* p<.05 . \quad * * p<.01 . \quad * * * p<.001$.

follow-up fatigue severity and disruptiveness among RT patients. After accounting for pretreatment levels, catastrophizing among RT patients accounted for $16 \%$ of the variability in fatigue severity $(p=.003)$ and $22 \%$ of the variability in fatigue disruptiveness at follow-up $(p=.0006)$.

\section{Discussion}

Results from the current study support the hypothesis that greater catastrophizing is related to greater fatigue in women undergoing treatment for breast cancer. This conclusion is qualified, however, by the presence of significant interaction effects between level of catastrophizing and type of treatment. Examination of these effects indicates that catastrophizing demonstrated expected relationships with fatigue severity and disruptiveness for RT patients but not for CT patients. Similarly, levels of catastroph- izing significantly predicted subsequent levels of fatigue severity and disruptiveness for RT patients but not for CT patients.

Prior to conducting the study analyses, we had speculated about possible interactive effects between type of treatment and level of catastrophizing. The observed Treatment $\times$ Catastrophizing interaction was consistent with the view that the impact of catastrophizing would be greater among patients receiving the less inherently fatiguing of the two treatments. Among patients receiving $\mathrm{RT}$ (the less fatiguing treatment), those high in catastrophizing reported significantly greater fatigue severity and disruptiveness than those low in catastrophizing. Among patients receiving CT (the more fatiguing treatment), levels of fatigue severity and disruptiveness were found to be similar in patients high and low in catastrophizing. The level of fatigue severity reported by the average CT patient following the start of chemotherapy can be characterized as moderate and is sim-
Radiotherapy

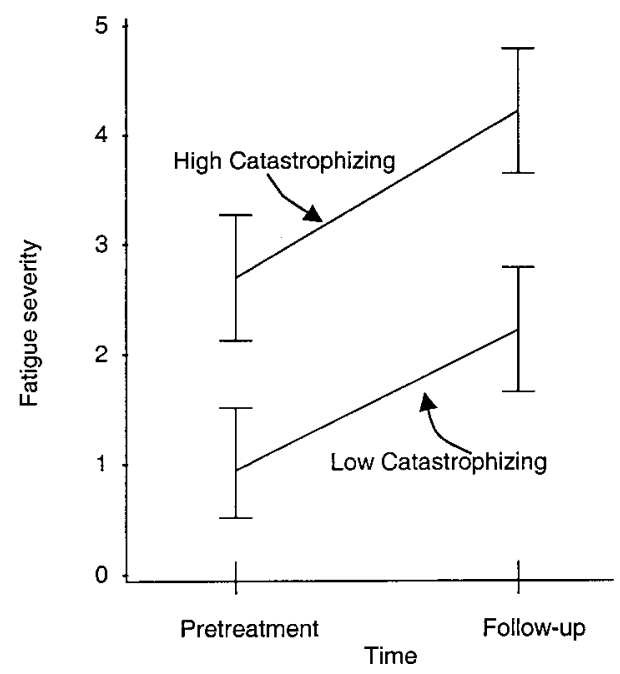

Chemotherapy

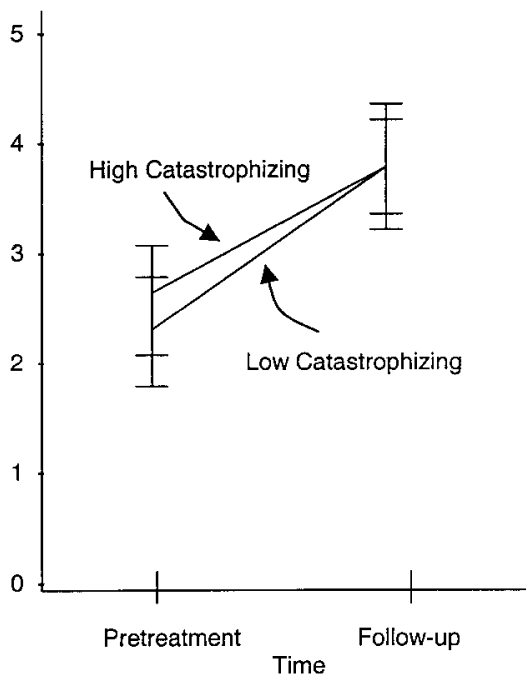

Figure 1. Relationship of level of catastrophizing and type of cancer treatment to fatigue severity. 
Table 4

Repeated Measures Analysis of Variance of Fatigue Disruptiveness Scores

\begin{tabular}{lrrrr}
\hline \multicolumn{1}{c}{ Effect } & $d f$ & SS & MS & $F$ \\
\hline Between subjects & & & & \\
$\quad$ Treatment & 1 & 24.46 & 24.46 & $6.65^{*}$ \\
Catastrophizing & 1 & 25.58 & 25.58 & $6.96^{*}$ \\
Treatment $\times$ Catastrophizing & 1 & 16.26 & 16.26 & $4.42^{*}$ \\
$\quad$ Residual & 75 & 275.87 & 3.68 & \\
Within subject & 1 & 38.26 & 38.26 & $18.70^{* * *}$ \\
Time & 1 & 0.00 & 0.00 & 0.00 \\
Time $\times$ Catastrophizing & 1 & 0.28 & 0.28 & 0.14 \\
Time $\times$ Treatment & 1 & 3.31 & 3.31 & 1.62 \\
Time $\times$ Catastrophizing $\times$ Treatment & 75 & 153.43 & 2.05 & \\
Residual & & & & \\
\hline
\end{tabular}

Note. $\mathrm{SS}=$ sums of squares; $\mathrm{MS}=$ mean square.

$* p<.05 . \quad * * * p<.001$

ilar to what we have observed previously in women receiving adjuvant chemotherapy for breast cancer (Jacobsen, Hann, et al., 1999). Among patients receiving RT, the magnitude of differences in fatigue between patients high and low in catastrophizing appeared to be substantial. Levels of fatigue severity and disruptiveness in RT patients high in catastrophizing were on a par with levels observed in CT. In contrast, levels of fatigue severity and disruptiveness among RT patients low in catastrophizing were significantly less than those reported by CT patients. Additional results regarding the relative importance of catastrophizing in predicting the subsequent experience of fatigue among $\mathrm{CT}$ and RT patients provided further evidence of treatment-related differences in the impact of catastrophizing.

These findings underscore the importance of considering treatment factors in attempts to understand psychological influences on symptom reporting. A similar pattern of results has been reported in research on the contribution of anxiety to reports of posttreatment nausea in patients receiving $\mathrm{CT}$ for cancer. $\mathrm{CT}$ regimens are known to differ considerably in their pharmacological potential to produce nausea and vomiting (Hesketh et al., 1997). On the basis of these considerations, Andrykowski and Gregg (1992) hypothesized that anxiety would have a greater impact on the experience of posttreatment nausea among patients receiving regimens with low to moderate emetic potential than it would on patients receiving regimens with high emetic potential. With increasing emetic potential, the impact of pharmacological variables would presumably override the contribution of psychological variables. Consistent with predictions, results showed that the strength of the relationship between anxiety and posttreatment nausea was inversely related to the emetic potential of the $\mathrm{CT}$ regimen that patients received. Preliminary evidence suggests that, in addition to their emetic potential, CT regimens differ in their fatigueinducing potential (von Pawel et al., 1999). If confirmed, then studies seeking to examine the relationship of psychological variables to chemotherapy-related fatigue should include some consideration of the fatigue-inducing potential of the regimens administered. The present study did not provide an opportunity to examine this issue, as all but 1 patient in the CT group received regimens containing cyclophosphamide and doxorubicin.

The conclusion that differences in the impact of catastrophizing were attributable to differences in the type of treatment must be considered provisional in light of the fact that patients were not randomized to receive CT or RT. Indeed, the two treatment groups differed on several demographic and clinical variables, including age, type of surgery, and disease stage. Two features should be noted in this regard. First, differences on these variables are not unexpected in light of current medical consensus (National Institutes of Health Consensus Development Panel, 2001) and current patterns of clinical practice (Hebert-Croteau, Brisson, Latreille, Blanchette, \& Deschenes, 1999). Second, none of these variables was related significantly to catastrophizing or to fatigue severity and disruptiveness in the present study. These features increase confidence that the observed interactions between catastrophizing and type of treatment are not confounded by relationships with other variables.

The conclusion that differences in fatigue among RT patients are specific to catastrophizing must also be considered provisional. In both clinical and experimental research on pain, catastrophizing has been shown to be significantly correlated with depression (Sullivan, Bishop, \& Pivik, 1995; Sullivan, Stanish, Waite, Sullivan, \& Tripp, 1998). Although several studies indicate that the relation between catastrophizing and pain is independent of depression (Sullivan et al., 1998; Walsh, Smith, \& McGrath, 1998), this issue has yet to be addressed for fatigue. In addition, it should be noted that we did not assess what information patients may have received about fatigue prior to the start of radiotherapy. In light of findings demonstrating that patient expectations explain differences in the experience of other cancer treatment side effects, such as chemotherapy-related nausea (Montgomery \& Bovbjerg, 2000; Roscoe, Hickok, \& Morrow, 2000), this issue merits careful consideration.

Findings from the present study suggest several directions for future research. One direction would be to determine whether catastrophizing about fatigue during the active treatment period is a risk factor for persistent fatigue following treatment completion. Although several studies have documented heightened fatigue in women previously treated with CT or RT for early stage breast cancer (Andrykowski, Curran, \& Lighter, 1998; Broeckel et al., 1998), the mechanisms underlying this "off-treatment" fatigue remain obscure. Research on chronic fatigue in other medically ill populations suggests that catastrophizing might promote behaviors (such as avoidance of physical activity) that could perpetuate 
fatigue independent of the original precipitating stimulus (Wessely, Hotopf, \& Sharpe, 1998).

Another future direction would be to determine whether current findings are generalizable to other populations of cancer patients. In particular, it would be important to learn about catastrophizing and its relationship to fatigue in male cancer patients, as all previous research on this topic has been conducted with female cancer patients. The possible presence of gender differences is supported by research on catastrophizing in relation to pain. Several studies have found that women score higher than men on measures of catastrophizing and that these differences mediate observed gender differences in pain reports and pain behavior (Keefe et al., 2000; Sullivan, Tripp, \& Santor, 2000).

Results regarding catastrophizing also provide support for developing a cognitive-behavioral model of fatigue in cancer patients. Research on chronic pain suggests that certain behaviors, such as activity reduction (Turner \& Clancy, 1986) and solicitous actions on the part of others (Sullivan et al., 2000), are related to catastrophizing and may serve to further exacerbate the pain experience. Greater inactivity has been shown to be associated with greater fatigue in women being treated for breast cancer (Berger, 1998); however, the relationship of inactivity to catastrophizing has yet to be investigated in cancer patients.

Still another future direction would be to determine the extent to which catastrophizing represents a general versus a specific response to the experience of symptoms. Research indicates that cancer patients typically experience multiple symptoms (such as nausea, pain, and fatigue) concurrently during the course of their treatment (Dodd, Miaskowski, \& Paul, 2001). This situation provides a relatively unique opportunity to examine whether catastrophizing occurs on a symptom-specific basis or whether it represents a more generalized response to aversive symptoms. If the latter is true, it would suggest that catastrophizing may have a broad impact on quality of life during cancer treatment.

Finally, results of the current study provide support for exploring new ways to intervene to reduce fatigue in women undergoing treatment for early stage breast cancer. Previous research has demonstrated that interventions based on cognitive-behavioral models can lead to reductions in catastrophizing that, in turn, are associated with better adjustment to chronic pain (Jensen, Turner, $\&$ Romano, 2001). These findings raise the possibility that similar interventions may be effective against fatigue, particularly in women receiving RT for breast cancer.

In conclusion, the present study demonstrates the importance of considering treatment factors in attempts to understand psychological influences on symptom reports. Findings showing relationships between catastrophizing and fatigue among RT patients but not among CT patients are consistent with the view that as the inherent symptom-inducing potential of treatment decreases, psychological factors play a greater role in patients' experience of symptoms. The current findings also extend our understanding of catastrophizing beyond its previously studied relationship to the experience of pain. Differences in fatigue related to catastrophizing among RT patients appear to be substantial and should encourage further study of this phenomenon. In particular, it will be important to learn whether catastrophizing represents a general versus a specific response to the aversive consequences of cancer treatment and whether fatigue and other symptoms cancer patients experience can be relieved through the use of interventions designed to reduce catastrophizing.

\section{References}

Andrykowski, M. A., Curran, S. L., \& Lighter, R. (1998). Off-treatment fatigue in breast cancer survivors: A controlled comparison. Journal of Behavioral Medicine, 21, 1-18.

Andrykowski, M. A., \& Gregg, M. A. (1992). The role of psychological variables in post-chemotherapy nausea: Anxiety and expectation. Psychosomatic Medicine, 54, 48-58.

Berger, A. M. (1998). Patterns of fatigue and activity and rest during adjuvant breast cancer chemotherapy. Oncology Nursing Forum, 25, $51-62$.

Bower, J. E., Ganz, P. A., Desmond, K. A., Rowland, J. H., Meyerowitz, B. E., \& Belin, T. R. (2000). Fatigue in breast cancer survivors: Occurrence, correlates, and impact on quality of life. Journal of Clinical Oncology, 18, 743-753.

Broeckel, J. A., Jacobsen, P. B., Horton, J., Balducci, L., \& Lyman, G. H. (1998). Characteristics and correlates of fatigue after adjuvant chemotherapy for breast cancer. Journal of Clinical Oncology, 16, 1689-1696.

Dodd, M. J., Miaskowski, C., \& Paul, S. M. (2001). Symptom clusters and their effect on functional status of patients with cancer. Oncology Nursing Forum, 28, 465-470.

Geinetz, H., Zimmerman, F. B., Stoll, P., Thamm, R., Kaffengerger, W., Ansorg, K., et al. (2001). Fatigue, serum cytokine levels, and blood cell counts during radiotherapy of patients with breast cancer. International Journal of Radiation Oncology, Biology, and Physics, 51, 691-698.

Greene, D., Nail, L. M., Fieler, V. K., Dudgeon, D., \& Jones, L. S. (1994). A comparison of patient-reported side effects among three chemotherapy regimens for breast cancer. Cancer Practice, 2, 57-62.

Hann, D. M., Jacobsen, P. B., Azzarello, L. M., Martin, S. C., Curran, S. L., Fields, K. K., et al. (1998). Measurement of fatigue in cancer patients: Development and validation of the Fatigue Symptom Inventory. Quality of Life Research, 7, 301-310.

Hebert-Croteau, N., Brisson, J., Latreille, J., Blanchette, C., \& Deschenes, L. (1999). Compliance with consensus recommendations for the treatment of early stage breast carcinoma in elderly women. Cancer, 85, 1104-1113.

Hesketh, P. J., Kris, M. G., Grunberg, S. M., Beck, T., Hainsworth, J. D., Harker, G., et al. (1997). Proposal for classifying the acute emetogenicity of cancer chemotherapy. Journal of Clinical Oncology, 15, 103-109.

Irvine, D. M., Vincent, L., Graydon, J. E., \& Bubela, N. (1998). Fatigue in women with breast cancer receiving radiation therapy. Cancer Nursing, $21,127-135$.

Jacobsen, P. B., Azzarello, L. M., \& Hann, D. M. (1999). Relation of catastrophizing to fatigue severity in women with breast cancer. Cancer Research, Therapy, and Control, 8, 155-164.

Jacobsen, P. B., Hann, D. M., Azzarello, L. M., Horton, J., Balducci, L., \& Lyman, G. H. (1999). Fatigue in women receiving adjuvant chemotherapy for breast cancer: Characteristics, course, and correlates. Journal of Pain and Symptom Management, 18, 233-242.

Jensen, M. P., Turner, J. A., \& Romano, J. M. (2001). Changes in pain beliefs, catastrophizing, and coping are associated with improvement in multidisciplinary pain treatment. Journal of Consulting and Clinical Psychology, 69, 655-662.

Keefe, F. J., Lefebvre, J. C., Egert, J. R., Affleck, G., Sullivan, M. J., \& Caldwell, D. S. (2000). The relationship of gender to pain, pain behavior and disability in osteoarthritis patients: The role of catastrophizing. Pain, 87, 325-334.

Longman, A., Braden, C. J., \& Mishel, M. H. (1996). Side effects burden in women with breast cancer. Cancer Practice, 4, 274-280.

Montgomery, G. H., \& Bovbjerg, D. H. (2000). Pre-infusion expectations predict post-treatment nausea during repeated adjuvant chemotherapy 
infusions for breast cancer. British Journal of Health Psychology, 5, $105-119$.

National Institutes of Health Consensus Development Panel. (2001). National Institutes of Health Consensus Development Conference statement: Adjuvant therapy for breast cancer, November 1-3, 2000. Journal of the National Cancer Institute Monographs, 30, 5-15.

Roscoe, J. A., Hickok, J. T., \& Morrow, G. R. (2000). Patient expectations as predictor of chemotherapy-induced nausea. Annals of Behavioral Medicine, 22, 121-126.

Smets, E. M. A., Visser, M. R. M., Willems-Groot, A. F. M. N., Garssen, B., Oldenburger, F., van Tienhoven, G., et al. (1998). Fatigue and radiotherapy: (A) Experience in patients undergoing treatment. British Journal of Cancer, 78, 899-906.

Sullivan, M. J. L., Bishop, S., \& Pivik, J. (1995). The Pain Catastrophizing Scale: Development and validation. Psychological Assessment, 7, 524532 .

Sullivan, M. J. L., \& D'Eon, J. (1990). Relation between catastrophizing and depression in chronic pain patients. Journal of Abnormal Psychology, 99, 260-263.

Sullivan, M. J. L., Stanish, W., Waite, H., Sullivan, M., \& Tripp, D. A. (1998). Catastrophizing, pain, and disability in patients with soft-tissue injuries. Pain, 77, 253-260.

Sullivan, M. J. L., Thorn, B., Haythornthwaite, J. A., Keefe, F., Martin, M., Bradley, L. A., et al. (2001). Theoretical perspectives on the relation between catastrophizing and pain. Clinical Journal of Pain, 17, 52-54.
Sullivan, M. J. L., Tripp, D. A., \& Santor, D. (2000). Gender differences in pain and pain behavior: The role of catastrophizing. Cognitive Therapy and Research, 24, 121-134.

Turner, J. A., \& Clancy, S. (1986). Strategies for coping with chronic low back pain: Relationship to pain and disability. Pain, 24, 355-364.

von Pawel, J., Schiller, J. H., Shepherd, F. A., Fields, S. Z., Kleisbauer, J. P., Chrysson, N. G., et al. (1999). Topotecan versus cyclophosphamide, doxorubicin, and vincristine for the treatment of recurrent smallcell lung cancer. Journal of Clinical Oncology, 17, 658-667.

Walsh, T. M., Smith, C. P., \& McGrath, P. J. (1998). Pain correlates of depressed mood in young adults. Pain Research and Management, 3, $135-144$.

Wessely, S., Hotopf, M., \& Sharpe, M. (1998). Chronic fatigue and its syndromes. New York: Oxford University Press.

Winningham, M. L., Nail, L. M., Burke, M. B., Brophy, L., Cimprich, B., Jones, L. S., et al. (1994). Fatigue and the cancer experience: The state of the knowledge. Oncology Nursing Forum, 21, 23-36.

Woo, B., Dibble, S. L., Piper, B. F., Keating, S. B., \& Weiss, M. C. (1998). Differences in fatigue by treatment methods in women with breast cancer. Oncology Nursing Forum, 25, 915-920.

Received December 5, 2002

Revision received June 24, 2003

Accepted July 2, 2003 